\title{
Competitive inter-species interactions underlie the increased antimicrobial tolerance in multispecies brewery biofilms
}

\author{
Ilse Parijs ${ }^{1} \cdot$ Hans P. Steenackers ${ }^{1}$
}

Received: 3 August 2017 / Revised: 17 March 2018 / Accepted: 20 March 2018 / Published online: 1 June 2018

(c) International Society for Microbial Ecology 2018

\begin{abstract}
Genetic diversity often enhances the tolerance of microbial communities against antimicrobial treatment. However the sociobiology underlying this antimicrobial tolerance remains largely unexplored. Here we analyze how inter-species interactions can increase antimicrobial tolerance. We apply our approach to 17 industrially relevant multispecies biofilm models, based on species isolated from 58 contaminating biofilms in three breweries. Sulfathiazole was used as antimicrobial agent because it showed the highest activity out of 22 biofilm inhibitors tested. Our analysis reveals that competitive interactions dominate among species within brewery biofilms. We show that antimicrobial treatment can reduce the level of competition and therefore cause a subset of species to bloom. The result is a 1.2-42.7-fold lower percentage inhibition of these species and increased overall tolerance. In addition, we show that the presence of Raoultella can also directly enhance the inherent tolerance of Pseudomonas to antimicrobial treatment, either because the species protect each other or because they induce specific tolerance phenotypes as a response to competitors. Overall, our study emphasizes that the dominance of competitive interactions is central to the enhanced antimicrobial tolerance of the multispecies biofilms, and that the activity of antimicrobials against multispecies biofilms cannot be predicted based on their effect against monocultures.
\end{abstract}

\section{Introduction}

Microbes commonly live in surface-attached communities embedded in a self-produced matrix, known as biofilms, which cause major problems and economic losses within industrial and medical sectors [1]. The majority of natural biofilms contain multiple species and harbor different functions and abilities compared to their monospecies counterparts $[2,3]$. One hallmark of multispecies biofilms is their increased tolerance against antimicrobial agents [4-19]. Although different species within biofilms are closely associated and are expected to strongly interact with each other [2], little is known about how these interactions affect

Electronic supplementary material The online version of this article (https://doi.org/10.1038/s41396-018-0146-5) contains supplementary material, which is available to authorized users.

$\triangle$ Hans P. Steenackers

hans.steenackers@kuleuven.be

1 Department of Microbial and Molecular Systems, Centre of Microbial and Plant Genetics (CMPG), KU Leuven, Kasteelpark Arenberg 20 - box 2460, B-3001 Leuven, Belgium antimicrobial tolerance. Indeed, most previous studies focused on the overall tolerance of multispecies biofilms, without looking at the contributions of individual species. In the limited cases that multispecies composition before and after treatment was determined, the types of interactions and their interdependency with antimicrobial treatment and tolerance were generally not investigated $[5,9,11,13,16-19]$.

Interactions can be cooperative or competitive in nature. Cooperative interactions for example involve the secretion of enzymes [20] or metabolic cross-feeding [21]. Social evolution theory defines a cooperative adaptation in one species as a phenotype that increases the fitness of another species and that evolved at least in part because of this effect [22-24]. This implies that both species are benefitting from the interaction, since it is difficult to see how an adaptation that helps another species can evolve when it has a fitness cost to the helping species. The cooperation criterion, therefore, states that an observed interaction is only consistent with a cooperative adaptation if the total productivity in coculture is higher than the sum of the monoculture productivities (which is the null for no interaction) and both species increase their cellular productivity in coculture vs. monoculture [23]. Higher order cooperative interactions could potentially occur when sets of three, four, 
or more species engage in loops of mutually beneficial interactions. In addition to evolved cooperation, accidental positive effects can occur (no positive feedback loop), for example, when waste products in a focal species can be used as resource by another species. This can result in a commensal interaction, in which there is no fitness effect in one direction, or can happen within a competitive association (vide infra).

Whenever one or both species experience a disadvantage in coculture, competition is dominant. Competition is expected to be favored when coexisting species have overlapping metabolic niches, are spatially mixed and when cell density is high relative to the available resources [25]. Phenotypes involved in microbial competition can be accidental in nature or have evolved for this purpose. The latter phenotypes are called competitive adaptations and include (i) strategies to take away resources from competitors (exploitative competition), for example by fast-butwasteful-growth [26], production of nutrient-scavenging molecules [27], or superior positioning within the niche [28], and (ii) strategies to directly fight with competing species (interference competition), for example by production of antimicrobials [29] or contact dependent inhibition $[30,31]$. Competition can be further characterized by comparing the observed productivity in coculture with the weighted average productivity of the constituent species in monoculture. This allows to determine to which extent inter-species competition differs from intra-specific competition. Frequently applied in plant ecology, this difference is called the biodiversity effect and is constituted of a selection effect and complementarity effect [32-36].

Here we combine the cooperation criterion and biodiversity effect to investigate how inter-species interactions underlie the increased tolerance of multispecies biofilms. We apply our approach to industrially relevant multispecies biofilm models consisting of combinations of species isolated from contaminating biofilms in breweries. In the brewing industry undesired biofilms can be associated with spoilage organisms, cause corrosion and reduce process efficiency. Several stages of the brewing process, including pasteurization, storage, and bottling of the beer, are known to be affected by biofilms and the eradication of these biofilms remains highly challenging [37-39]. Improved understanding of antimicrobial tolerance is thus a prerequisite for designing more effective brewery sanitation procedures.

Our analysis reveals that a complex interplay between antimicrobial treatment and genetic diversity underlies the commonly observed increased tolerance of multispecies biofilms. Consistent with previous work in other microbial communities [22, 40, 41], we show that competitive interactions dominate among species within the brewery biofilms. We then show that this dominance of competitive interactions is central to the enhanced antimicrobial tolerance of the multispecies biofilms. Specifically we found that strongly suppressed species can bloom if antimicrobial treatment inhibits superior competitors. The result is an increased tolerance of these blooming species in the multispecies biofilm compared to the monoculture biofilms, even when the activity of the antimicrobial is equal in monoculture and coculture. Complete inhibition of all species in the mixture would avoid this effect. However, our results further indicate that the presence of competing species can also directly enhance the inherent tolerance of microbes to antimicrobial treatment. Antimicrobials that are completely effective against monoculture biofilms are thus not necessarily effective against the same species in coculture. Overall this emphasizes that the activity of antimicrobials against multispecies biofilms cannot be predicted based on their effect against monocultures. Alternation between different antimicrobials that target different species could provide a strategy to avoid enrichment of specific species. This should be combined with strategies that interfere with the mechanisms behind specific tolerance phenotypes driven by the presence of competitors.

\section{Material and methods}

\section{Sampling}

A total of 58 samples were collected from four breweries in Belgium between August and December 2014, before and after cleaning in place (CIP). The CIP protocol generally starts with mechanical cleaning by rinsing with (hot) water. Next, a caustic or an acidic agent is used to chemically remove contaminations. Finally in some cases a disinfectant is added to target microbial contamination [42, 43]. Samples taken before CIP were collected at least $12 \mathrm{~h}$ after the last cleaning, whereas the samples taken after CIP were collected within $3 \mathrm{~h}$ after cleaning. The sampling protocol is similar to protocols used by other studies that isolated brewery biofilms [37, 38]. Sterile cotton swabs (Deltalab) were used for collection of biofilm material from approximately $25 \mathrm{~cm}^{2}$ on different surfaces in the bottling plant, filtration room, and storage room. After sampling, swabs were submerged in phosphate buffered saline (PBS), which consisted of $8.8 \mathrm{~g} / \mathrm{L} \mathrm{NaCl}, 1.24 \mathrm{~g} / \mathrm{L} \mathrm{K} \mathrm{HPO}_{4}$, and $0.39 \mathrm{~g} / \mathrm{L}$ $\mathrm{KH}_{2} \mathrm{PO}_{4}$ (pH 7.4). Biofilm material was removed from the swabs by $3 \times 30 \mathrm{~s}$ vortexing and sonication at $45 \mathrm{kHz}, 80 \mathrm{~W}$. Nine hundred microlitres of the PBS solution with the biofilm material was frozen at $-80{ }^{\circ} \mathrm{C}$ in $50 \%$ glycerol. One hundred microlires was plated out in dilution $10^{0}-10^{-8}$ on plate count agar (PCA), composed of $5 \mathrm{~g} / \mathrm{L}$ peptone, $2.5 \mathrm{~g} / \mathrm{L}$ yeast extract, $1 \mathrm{~g} / \mathrm{L}$ glucose, and $15 \mathrm{~g} / \mathrm{L}$ agar. The plates 
were incubated at $25{ }^{\circ} \mathrm{C}$ for 7 days. The total culturable microbial load was determined by counting the colonies on the plates that contained between 30 and 300 colonies. The $\mathrm{CFU} / \mathrm{cm}^{2}$ was determined based on the number of colonies in the $100 \mu$ liquid that was plated out and the surface of the well $\left(0.36 \mathrm{~cm}^{2}\right)$.

\section{Identification of culturable species}

Colonies growing on the PCA plates were identified by partial 16S rRNA or ITS gene sequencing. For bacteria, colony PCR using Taq DNA polymerase (Life Technologies) was performed on the 16S rRNA gene, which was targeted by primers BSF8/20 and BSR $1541 / 20$ [44]. The following PCR program was used: $96^{\circ} \mathrm{C}$ for $6 \mathrm{~min}, 35$ cycles of (i) denaturation at $96^{\circ} \mathrm{C}$ for $1 \mathrm{~min}$, (ii) annealing at $47.5^{\circ} \mathrm{C}$ for $1 \mathrm{~min}$, and (iii) elongation at $72^{\circ} \mathrm{C}$ for $90 \mathrm{~s}$, and a final elongation at $72{ }^{\circ} \mathrm{C}$ for $6 \mathrm{~min}$. Afterward, the PCR amplicons were loaded on an agarose $(10 \mathrm{~g} / \mathrm{L})$ gel, which was run for $1 \mathrm{~h}$ at $125 \mathrm{~V}$ and $400 \mathrm{~A}$. The band at $1500 \mathrm{bp}$ was cut out and the DNA was extracted by using the GenElute $^{\mathrm{TM}}$ Gel Extraction Kit (Sigma-Aldrich). Sanger sequencing was performed on the extracted DNA using primer BSF8/20 (GATC Biotech). The resulting sequence was blasted against the NCBI gene database to identify the closest relative of each colony. For yeast identification, the same protocol was followed, using primers ITS1 and ITS4 [45], with an annealing temperature of $60^{\circ} \mathrm{C}$ and an elongation time of $1 \mathrm{~min}$. The resulting PCR fragment was 330 bp.

\section{Defined multispecies biofilm models}

Defined multispecies biofilm models combined a fixed number of culturable species that were isolated from the same sample. To this end, each species was grown in liquid PCA culture for $48 \mathrm{~h}$ at $25^{\circ} \mathrm{C}$ under shaking conditions. These species were combined with a starting density of $1000 \mathrm{CFU} / \mathrm{ml}$ for each species and grown in 1/ 20 Trypticase Soy Broth in 96-well plates. Similarly monospecies biofilms were set up with a starting density of $1000 \mathrm{CFU} / \mathrm{ml}$. After incubation for 4 days at $25^{\circ} \mathrm{C}$, the amount of living cells in the biofilm formed on the bottom of the 96-well plates was quantified by plate count. First, the free-living cells were removed from the wells. Second, the biofilm on the bottom of the wells was scraped off in $200 \mu \mathrm{l}$ PBS and diluted appropriately. Finally the diluted solutions with biofilm cells were plated out on PCA plates and incubated at $25^{\circ} \mathrm{C}$ for $2-5$ days. Colonies on the plates were counted and the $\mathrm{CFU} / \mathrm{cm}^{2}$ was determined as previously described. In the multispecies biofilms, a distinction was made between the different species based on colony morphology.

\section{Study of inter-species interactions: cooperation criterion and biodiversity effect}

Fifteen different defined multispecies biofilms were grown as described above. To determine if inter-species interactions were cooperative or competitive, the cooperation criterion was applied. This criterion requires that the biofilm growth for all species in coculture is higher than their respective biofilm growth in monoculture [23].

To further characterize the ecological influences on interactions, the biodiversity effect was calculated according to the formula below, in which $N \overline{\Delta R Y M}$ measures the complementarity effect and $N \operatorname{cov}(\Delta R Y, M)$ measures the selection effect [33].

$$
\Delta Y=Y_{O}-Y_{E}=N \overline{\Delta R Y \bar{M}}+N \operatorname{cov}(\Delta R Y, M)
$$

$M_{i}=$ biofilm growth of species i in monoculture

$Y_{O, i}=$ observed biofilm growth of species i in coculture

$Y_{O}=\sum_{i} Y_{O, i}=$ total observed biofilm growth in coculture

$R Y_{E, i}=$ expected relative biofilm growth of species $\mathrm{i}$ in coculture, which is its proportion inoculated

$R Y_{O, i}=Y_{O, i} / M_{i}=$ observed relative biofilm growth of species i in coculture

$Y_{E, i}=R Y_{E, i} M_{i}=$ expected biofilm growth of species $\mathrm{i}$ in coculture

$Y_{E}=\sum_{i} Y_{E, i}=$ total expected biofilm growth in coculture

$\Delta Y=Y_{O}-Y_{E}=$ deviation from the total expected biofilm growth in the coculture (=biodiversity effect)

$\Delta R Y_{i}=R Y_{O, i}-R Y_{E, i}=$ deviation from expected relative biofilm growth of species $i$ in coculture

$N=$ number of species in coculture

The cooperation criterion requires the total multispecies inoculation density to be equal to the sum of the inoculation densities of the monoculture biofilm. In contrast, the definition of the biodiversity effect imposes that the inoculation density of each species in the multispecies biofilms should be its inoculation density in monoculture, divided by the number of species that are present in the multispecies biofilm. All multispecies biofilm models in this study were grown in both set ups and no differences in final growth were observed. Inoculation densities are indeed not expected to have large effects when biofilms are grown to stationary phase [22].

\section{Biofilm inhibitor screening}

A library of 96 inhibitors that were reported in literature or developed in house and that are known to affect biofilm specific processes was composed. Based on commercial availability, low cost, and toxicity, 22 compounds were selected for a one-replicate preventive screening against 17 
Fig. 1 Tolerance to cleaning in place (CIP) for 33 biofilms sampled from different locations in different breweries

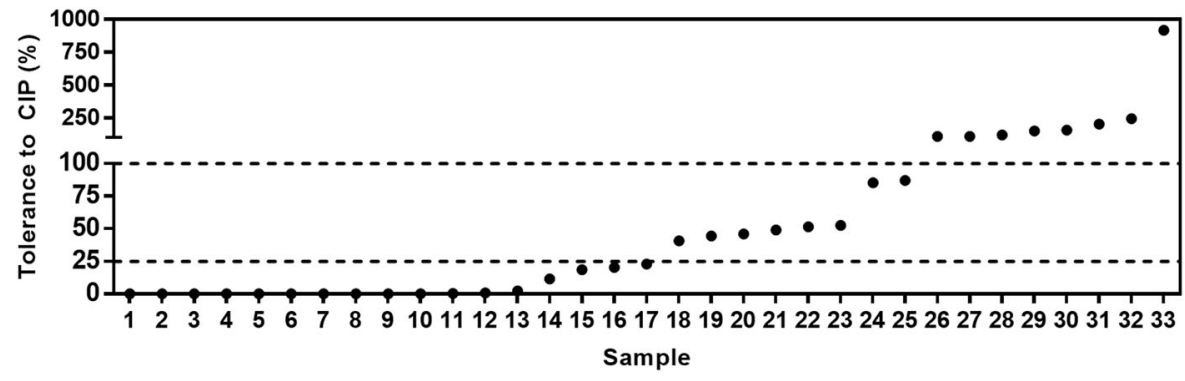

following formulas:

$\%$ Tolerance(species i, mono)

$$
\begin{gathered}
=\frac{\text { Treated biofilm growth }(\text { species } \mathrm{i}, \text { mono })}{\text { Untreated biofilm growth }(\text { species } i, \text { mono })} \times 100 \\
\% \text { Tolerance }(\text { species } i, \text { multi }) \\
=\frac{\text { Treated biofilm growth }(\text { species } i, \text { multi })}{\text { Untreated biofilm growth }(\text { species } i, \text { multi })} \times 100
\end{gathered}
$$

\section{Results}

\section{Construction of industrially relevant multispecies biofilm models}

We started by setting up a series of in vitro multispecies biofilm models with relevance for the brewing industry that were further used throughout this study. To this end, 103 biofilm samples isolated from different locations in several breweries were microbiologically characterized. The total bacterial load $\left(\mathrm{CFU} / \mathrm{cm}^{2}\right)$ varied between $10^{2}$ and $10^{8}$ before CIP and between $10^{1}$ and $10^{9}$ after CIP. As shown in Figure 1, the microbial contamination after CIP was reduced by less than $75 \%$ in $52 \%$ of the samples and was even increased in $24 \%$ of the samples, indicating that CIP is insufficient and that improved antimicrobial treatments are highly needed. The genera of the closest known relatives of the culturable microbes were determined by partial $16 \mathrm{~S}$ rRNA gene sequencing to characterize the microbial diversity (Table S1). The biofilm samples were mainly composed of Pseudomonas and Raoultella spp. and also two beer spoiling organisms, Pediococcus and Lactococcus, were identified. The different species were only identified to genus level but will be referred to as "species" further in the text. Duo-species (consisting of two species) and multispecies (consisting of more than two species) biofilm models were then constructed by combining species isolated from the same sample. Seventeen "undefined" multispecies biofilm models were set up by directly inoculating part of the frozen isolated biofilm samples. These models 

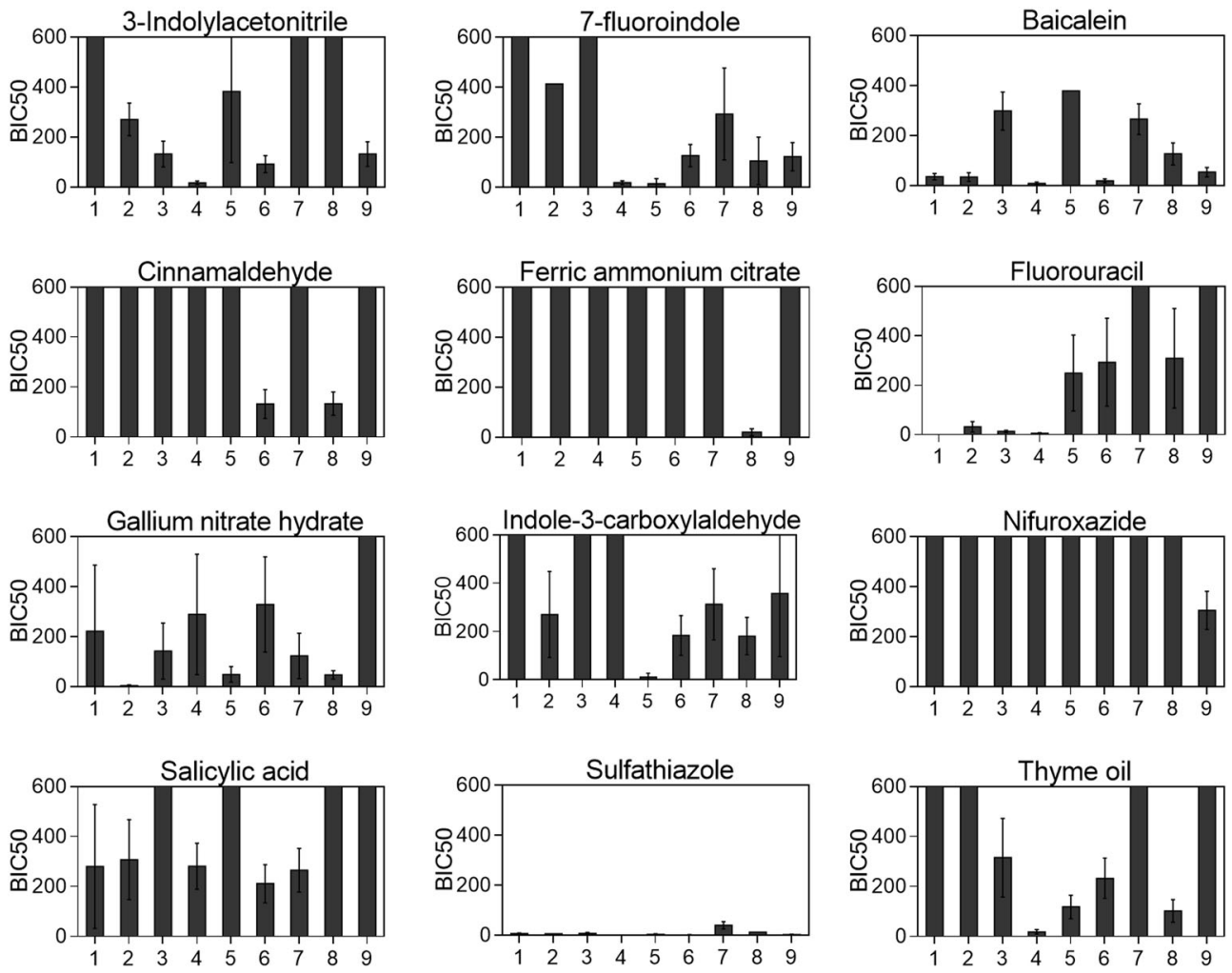

Fig. 2 The 50\% biofilm inhibitory concentration (BIC50) values of 12 biofilm inhibitors against 9 undefined biofilms (shown as mean with standard deviation of three biological replicates). BIC50 is defined as

the concentration $(\mu \mathrm{M})$ of inhibitor needed to prevent biofilm growth with $50 \%$. Compounds with BIC50 values over $600 \mu \mathrm{M}$ are considered ineffective and are not shown

were used to screen for broad-spectrum biofilm inhibitors. Because these biofilms likely contain unculturable species, for the study of inter-species interactions an additional 12 "defined" duo-species and multispecies biofilm models were constructed by inoculating equal ratios of 3-6 wellidentified, culturable species (originating from eight samples taken before CIP and four samples taken after CIP). Several biofilms containing Pseudomonas and Raoultella spp. were included.

\section{Screening of biofilm inhibitors}

To study the tolerance of multispecies brewery biofilms, we sought to use a broad-spectrum antimicrobial with potential for application against brewery biofilms. To this end, a library of 22 biofilm inhibitors, with previously reported activity against monoculture biofilms, was composed that target biofilm specific processes, such as adhesion [47], dispersion [48], EPS-production [49], and several other parameters [50]. After initial screening against 17 undefined multispecies biofilm models using a fixed concentration of $100 \mu \mathrm{M}$ (data not shown), we selected 12 inhibitors that were tested more thoroughly using multiple concentrations. Specifically we performed a preventive screening against 9 undefined multispecies biofilm models directly grown from the frozen brewery biofilm samples. Crystal violet staining was used to measure the amount of biofilm formed and the $50 \%$ inhibitory concentrations (BIC50) were calculated for each biofilm model (Fig. 2). Sulfathiazole was found to have the broadest activity spectrum against the brewery biofilms and was therefore selected for further study. This inhibitor has been described previously to interfere with cdi-GMP biosynthesis in E.coli biofilms [51]. C-di-GMP has been reported to play a crucial role in biofilm formation by a wide range of bacterial species, which might explain the broad-spectrum activity of this compound [52-54].

\section{Effect of inter-species interactions on biofilm growth and composition}

We first aimed to determine the role of inter-species interactions in multispecies biofilms, irrespective of antimicrobial treatment. To this end, we performed a systematic classification of the interactions in 12 defined biofilm models, each consisting of 3-6 culturable species, by using two complementary approaches: (i) cooperation criterion and (ii) biodiversity effect.

The cooperation criterion was used to classify interactions as cooperative or competitive. For all biofilm models, the number of biofilm cells of each species $\left(\mathrm{CFU} / \mathrm{cm}^{2}\right)$ in 
1

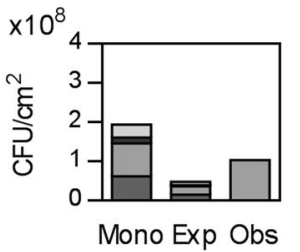

4

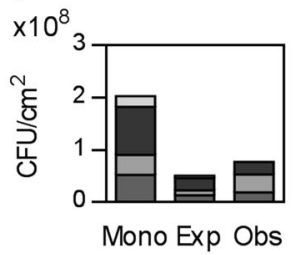

7

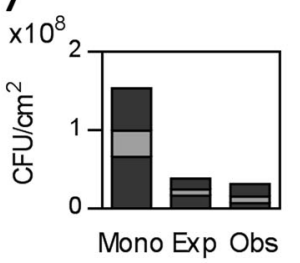

10
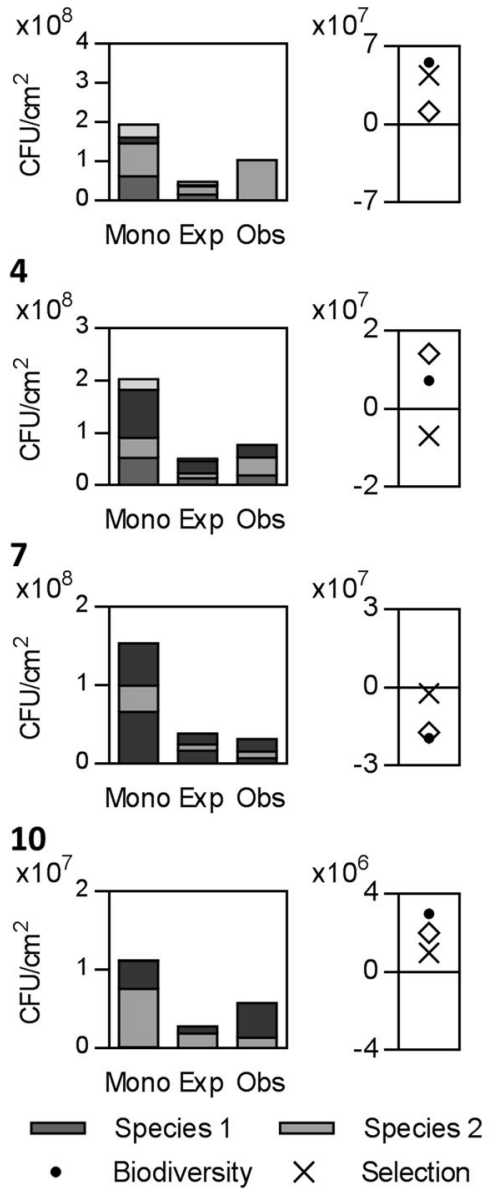

$\times 10^{7}$
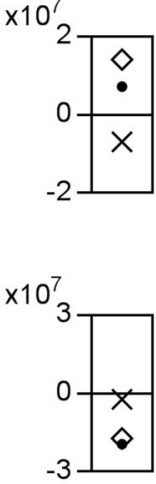

2

11

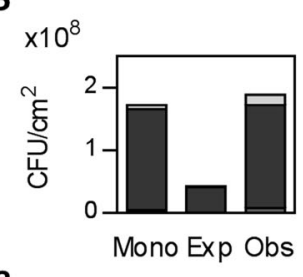

8
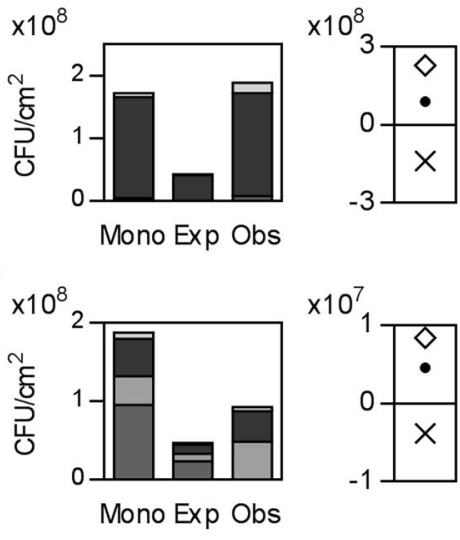

6
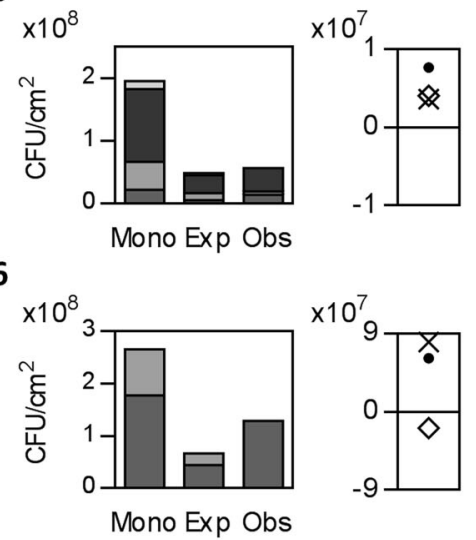

9
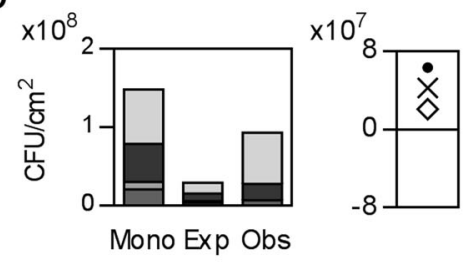

12
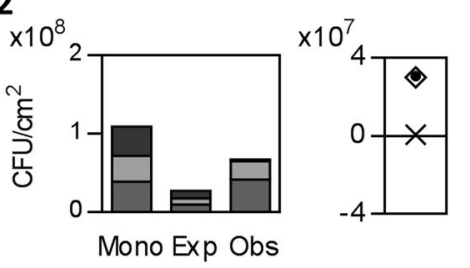

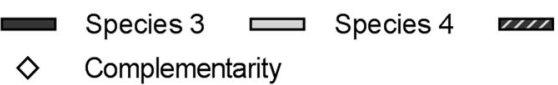

Fig. 3 Monoculture growth (mono), expected (exp), and observed (obs) multispecies composition and the biodiversity, selection, and complementarity effect for one representative replicate of 12 defined multispecies biofilm models

monoculture was compared to the cell count of each species in coculture (Fig. 3). Most species performed worse in coculture than in monoculture indicating that competitive interactions are dominant. The increased cellular productivity observed for a subset of the species in few of the multispecies biofilms (e.g., for 3 out of 4 species in model 5) could be due to exploitation of the remaining, suppressed species, however, cooperation between these species cannot be ruled out. In summary, all models were characterized by competitive interactions that cause some or all species to perform worse than in monoculture.

To further characterize competition, the biodiversity effect was measured [33]. When inter-species competition is equal to intra-specific competition, the observed productivity in coculture is expected to be equal to the average productivity of the constituent species in monoculture, weighted by the inoculation frequencies. The biodiversity effect is defined as the difference between the observed and expected multispecies biofilm productivity and is thus a measure for the extent to which inter-species interactions deviate from intra-specific interactions. The observed productivity of the 12 model biofilms $\left(\mathrm{CFU} / \mathrm{cm}^{2}\right)$ was compared to the expected biofilm growth (Fig. 3). In the majority of the multispecies biofilm models (75\%), the total amount of biofilm formed was higher than expected, as indicated by a positive biodiversity effect, whereas the remaining $25 \%$ of the cases were characterized by a negative biodiversity effect.

A positive biodiversity effect can either be caused by selection of the best biofilm former or by a (partial) niche separation alleviating competition; conversely a negative biodiversity effect can be caused by selection of the worse biofilm former or by strong interference competition. To distinguish between both possibilities, Loreau \& Hector [33] partitioned the biodiversity effect into a selection and complementarity effect (Material and methods). Selection occurs when the extent to which the relative productivity in coculture vs. monoculture deviates from expected is nonrandomly related to the productivity in monoculture and is measured by a covariance function. Positive selection is indicative of the dominance of the best monoculture biofilm formers and occurred in $33.3 \%$ of the multispecies biofilm 
Table 1 Closest known relative genus for each of the species present in the three multispecies biofilm models that were used to study the inhibition by sulfathiazole

\begin{tabular}{lllll}
\hline & Species 1 & Species 2 & Species 3 & Species 4 \\
\hline Model 1 & Epilithonimonas & Aeromonas & & \\
Model 2 & Pseudomonas & Pseudoclavibacter & Raoultella & Serratia \\
Model 3 & Pseudomonas & Raoultella & & \\
\hline
\end{tabular}

models. Negative selection suggests the opposite and appeared in the remaining $67.7 \%$ of the multispecies biofilm models. If only selection effects take place, the total relative productivity (sum of relative productivities of all species) is 1 , meaning that an increase in productivity in one species is compensated by a decrease in productivity of another species. However, if the total relative productivity is higher or lower than 1 overyielding or underyielding occurs, which is defined as the complementarity effect. This effect measures whether the relative amount of biofilm formed in coculture vs. monoculture is on average higher or lower than expected based on the initial relative abundance and biofilm growth in monoculture and is thus also a measure for the strength of competition. Complementarity is positive if some degree of niche separation occurs, for example if two species can grow on different resources or if one species is able to use a waste product of another species as a resource. Consequently the strength of competition decreases and the productivity increases due to a more optimal use of the available niches. Positive complementarity was observed in $91.7 \%$ of the multispecies biofilm models. On the other hand, negative complementarity effects occurred in the remaining $8.3 \%$ of the multispecies biofilm models and indicate the occurrence of strong chemical or physical interference competition [55-57].

The combination of complementarity and selection effects then gives an indication as to which ecological processes are the cause of the total positive or negative biodiversity effect. In our multispecies biofilm models (Fig. 3), positive biodiversity (75\%) could be explained by resource partitioning or facilitation between the different species for $66.7 \%$ of the biofilms (only positive complementarity), by dominance of the best biofilm formers for $11.1 \%$ of the biofilms (only positive selection), or by a combination of both positive complementarity and selection for $22.2 \%$ of the biofilms. Conversely negative biodiversity effects $(25 \%)$ were caused by exploitation or interference competition for $33.3 \%$ of the biofilms (only negative complementary), by dominance of poor biofilm formers for $33.3 \%$ of the biofilms (only negative selection), or by a combination of negative complementarity and selection for $33.3 \%$ of the biofilms [58]. Overall, the mainly positive complementarity effects indicate that the competitive interactions in the multispecies biofilm models are in most cases alleviated by partial ecological niche separation, in which species for example make use of different resources $[59,60]$.

\section{Link between reduced competition and antimicrobial tolerance in multispecies biofilms}

The results above show that competitive inter-species interactions, although in general alleviated by partial niche separation, strongly influence the productivity of each species in the multispecies biofilms. In a next step, we sought to investigate the interplay of these competitive interactions with antimicrobial treatment and their effect on antimicrobial tolerance. To this end, sulfathiazole was added preventively to three biofilm models (Table 1). Tolerance to sulfathiazole is defined as the ratio between the amount of biofilm formed in the presence and absence of treatment and was determined for each species in monoculture and coculture conditions (Figs. 4-6). In all three models the tolerance of each species was equal or higher in the multispecies biofilm than in the monoculture. The result was an overall increase in tolerance in each multispecies biofilm, which can be seen by comparing the expected and observed amount of biofilm after treatment. Here the expected amount was calculated based on the composition before treatment and the percentage of reduction of each species in monoculture. These results are consistent with the increased tolerance generally observed in multispecies biofilms [61, 62]. Specifically our results are consistent with a previous study on sulfathiazole treatment, in which multispecies biofilms isolated from cooling water systems were found to be more tolerant compared to their monoculture counterparts [14].

Analyzing biofilm compositions before and after treatment revealed that the above described dominance of competitive interactions in untreated biofilms is central to the observed enhanced tolerance to antimicrobial treatment. In two out of three biofilm models we found that antimicrobial treatment reduced the level of competition and therefore caused a subset of species to bloom. The result was a lower percentage inhibition of these species in the duo-species or multispecies biofilm compared to the monoculture biofilms, which-per definition-appears as increased tolerance.

In duo-species model 1 (Fig. 4), species 2 was sensitive to the inhibitor both in the monoculture and coculture 

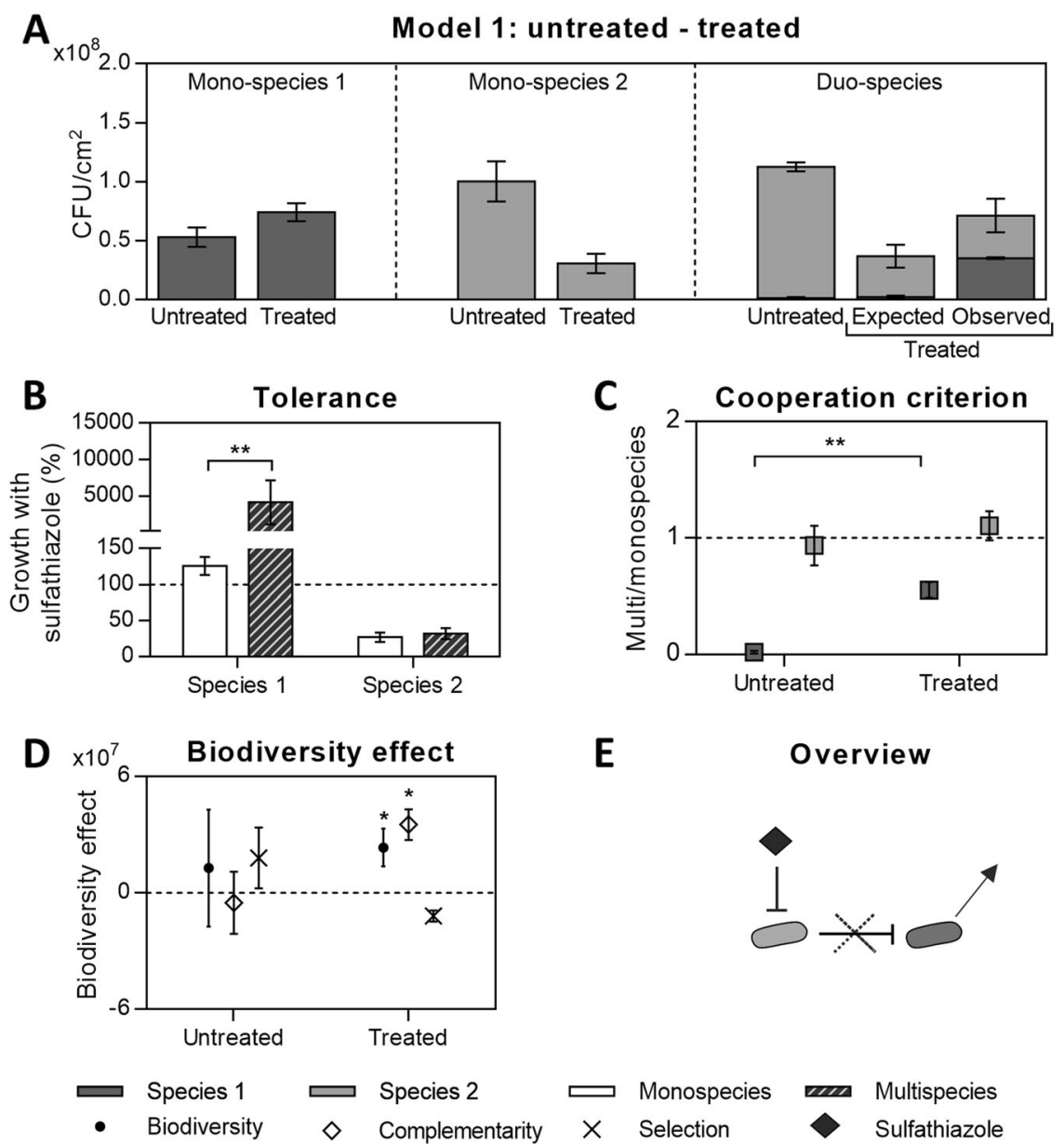

Fig. 4 Model 1: a Number of cells of each species in monoculture and coculture biofilms, grown in the absence and presence of sulfathiazole treatment: suppressed species 1 is able to grow after treatment. b Tolerance $=$ ratio between the number of biofilm cells with and without sulfathiazole treatment, determined for each species in monoculture and coculture conditions. For each species the tolerance is equal or higher within the coculture biofilm. c Cooperation: both in the absence and presence of treatment the criterion for cooperation is not met. Growth of species 1 in coculture increases after treatment, but does not exceed its growth in monoculture. d Biodiversity effects in the absence and presence of treatment of the duo-species biofilm:

biofilm (Fig. 4a, b). However, species 1, which was insensitive to the inhibitor in monoculture, showed a 50fold increase in growth upon addition of the inhibitor in the duo-species biofilm (Fig. 4a, b), resulting in an overall higher tolerance of the duo-species biofilm (Fig. 4a). In the untreated duo-species biofilm, species 1 was strongly suppressed by species 2 as reflected in the strong competition (Fig. 4c), large positive selection effect (Fig. 4d), and negative complementarity (Fig. 4d). The increased growth of species 1 upon treatment is therefore consistent with an abrogation of the competitive interactions of sensitive species 2 against species 1, which then blooms and shows a net increase in antimicrobial tolerance. This is dominating positive selection is replaced by positive complementarity after treatment. e Overview: inhibition of species 2 leads to a reduction in the competitive interactions against species 1 , which allows species 1 to bloom. Results show the average of three biological replicates, except for a, which shows the average of three technical replicates of one representative biological replicate. Error bars show the standard deviation of the repeats. Significant differences were examined using unpaired $t$-tests with Bonferroni correction for multiple comparisons $(* * P<0.01)$. Biodiversity effects that significantly differ from 0 were examined using a one-sample $t$-test $\left({ }^{*} P<0.05\right)$

reflected in a reduced competition (Fig. 4c), associated with a positive complementarity (Fig. 4d) in the treated biofilm. In summary, inhibition of the best competitor resulted in a bloom of the worse competitor and overall increased tolerance.

A similar mechanism played in tetra-species model 2 (Fig. 5). Three out of four species were completely inhibited both in the monospecies and multispecies biofilm, as measured by plating out of viable cells to dilution $10^{\circ}$ (Fig. 5a, b). Species 3, however, which was insensitive to the inhibitor in the monospecies biofilm, showed a 1.5-fold increase in growth upon addition of the inhibitor in the multispecies biofilm (Fig. 5a, b), resulting in an overall increase in 


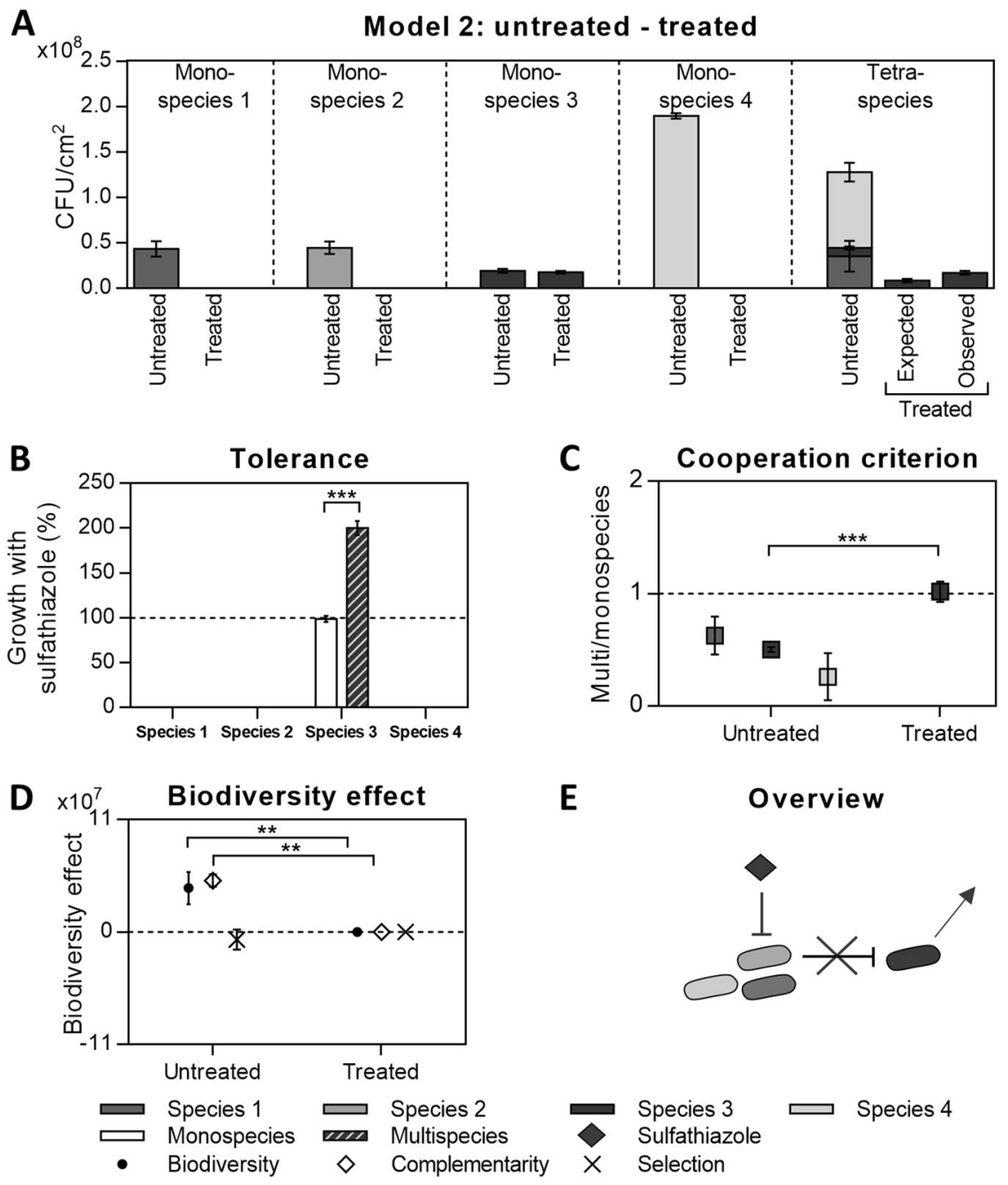

Fig. 5 Model 2: a Number of cells of each species in monoculture and coculture biofilms, grown in the absence and presence of sulfathiazole treatment: suppressed species 3 shows an increased growth upon treatment. b Tolerance $=$ ratio between the number of biofilm cells with and without sulfathiazole treatment, determined for each species in monoculture and coculture conditions. Species 3 shows an increased the tolerance within the multispecies biofilm, whereas species 1,2, and 4 are completely inhibited in monoculture and coculture. c Cooperation: both in the absence and presence of treatment the criterion for cooperation is not met. Growth of species 3 in coculture increases after treatment, but does not exceed its growth in monoculture. d

tolerance of the mixed species biofilm (Fig. 5a). Species 3 experienced competition by the other species in the untreated multispecies biofilm (Fig. 5c, d), explaining why inhibition of these other species increased the growth - and tolerance-of species 3 in the treated biofilm. Since there was only one species left after treatment, competition (Fig. 5c) and biodiversity effect (Fig. 5d) were zero.

It should be noted that this mechanism of "increased tolerance due to reduced competition" does not involve an increase in absolute cell numbers of the different species in treated coculture compared to treated monoculture, nor an
Biodiversity effects in the absence and presence of treatment of the multispecies biofilm: biodiversity effects disappear after treatment. e Overview: complete inhibition of species 1,2 , and 4 leads to the abrogation of competitive interactions against species 3 , which allows species 3 to bloom. Results show the average of three biological replicates, except a, which shows the average of three technical replicates of one representative biological replicate. Error bars show the standard deviation of the repeats. Significant differences were examined using unpaired $t$-tests with Bonferroni correction for multiple comparisons $(* * P<0.01, * * * P<0.001)$

expression of specific tolerance phenotypes. Nevertheless, the proposed mechanism is of significance. Indeed, similar to our study, antimicrobial tolerance in previous studies was generally measured by calculating the reduction in cell numbers before and after treatment, not by directly comparing the absolute cell numbers between coculture and monoculture conditions [17, 18, 63-65]. Therefore, the increased tolerance observed in these studies might as well be explained by decreased competition and should not necessarily be accompanied by any changes in specific tolerance phenotypes. 


\section{Direct effect of competitors on antimicrobial tolerance}

The findings above indicate that incomplete antimicrobial treatment of multispecies biofilms can reduce the levels of competition and therefore cause a subset of species to bloom, which ultimately results in increased antimicrobial tolerance. Complete inhibition of all species in the mixture would solve this problem. However, our analysis of duospecies model 3 (Fig. 6) indicated that the presence of competing species could also directly enhance the inherent tolerance of other species by driving specific tolerance phenotypes. This means that antimicrobials that are completely effective against monoculture biofilms are not necessarily effective against the same species in coculture and thus precludes any prediction on multispecies tolerance.

In this duo-species model (Fig. 6), both species responded to sulfathiazole treatment in the monoculture and coculture biofilms (Fig. 6a, b). However, species 1 showed a 11.1-fold reduction in sensitivity in coculture, resulting in an overall increased tolerance of the coculture biofilm (Fig. 6a). In contrast to the previous model systems, this tolerance of species 1 was associated with an increase in cell number above the treated monoculture level (Fig. 6a, right panel). These results could not be explained by a decrease in competition alone (Fig. 6c, d), and should be attributed to the presence of specific tolerance phenotypes within the multispecies biofilm. These could either be related to a
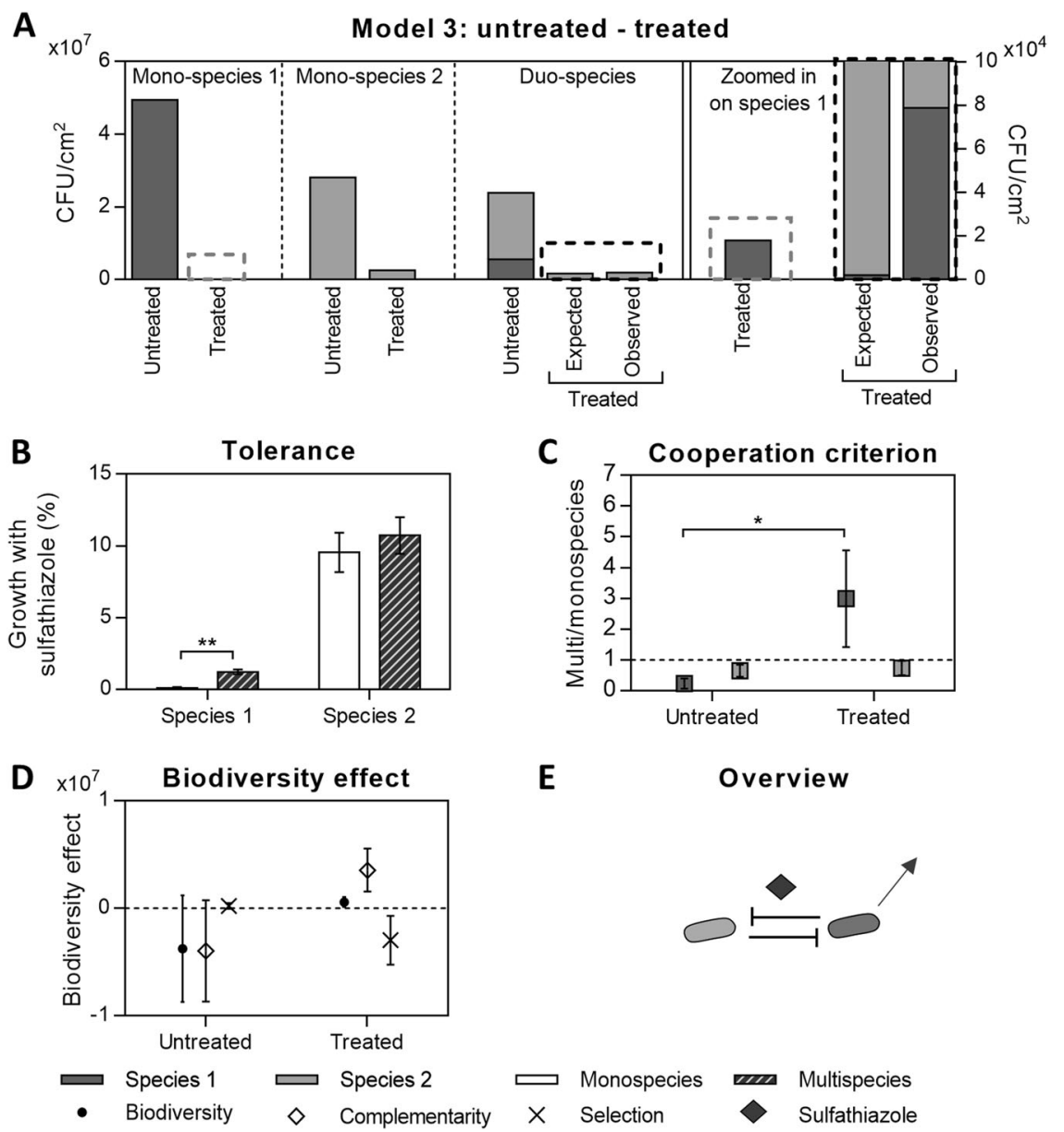

Fig. 6 Model 3: a Number of cells of each species in monoculture and coculture biofilms, grown in the absence and presence of sulfathiazole treatment. The right part of the graph zooms in on the amount of biofilm formed by species 1 in treated monoculture and duo-culture: after treatment the growth of species 1 in duo-culture exceeds its growth in monoculture. $\mathbf{b}$ Tolerance $=$ ratio between the number of biofilm cells with and without sulfathiazole treatment, determined for each species in monoculture and coculture conditions. Species 1 shows a higher tolerance within the coculture biofilm, whereas there is no difference for species 2. c Cooperation: after treatment species 1 grows better in coculture than in monoculture, whereas there is no difference

for species 2. This is consistent with commensalism. d Biodiversity effects in the absence and presence of treatment of the coculture biofilm: negative complementarity before treatment becomes positive. e Overview: species 1 becomes more tolerant in the presence of competing species 2 . The growth of species 1 in the treated coculture biofilm even exceeds its monoculture growth, suggesting induction of specific tolerance phenotypes. Results show the average of three biological replicates, except $\mathbf{a}$, which shows one representative biological replicate. Error bars show the standard deviation of the repeats. Significant differences were examined using unpaired $t$-tests with Bonferroni correction for multiple comparisons $(* P<0.05, * * P<0.01)$ 
protective effect of species 2 on species 1 or to a direct change in tolerance phenotype of species 1 as a response to species 2 .

\section{Discussion}

Functional properties like antimicrobial tolerance strongly differ between multispecies and monospecies biofilm communities [62, 66]. Although inter-species interactions are expected to be both intense and important within dense communities [2], little is known about how they affect antimicrobial tolerance. Previous work either focused on microbial interactions in untreated biofilms $[25,66,67]$ or on the overall tolerance of multispecies biofilms, without taking contributions of individual species into account [4, $10,12,15,68]$. We have bridged the gap and shown that a complex interplay between antimicrobial treatment and inter-species interactions underlies the commonly observed increased tolerance of multispecies biofilms. We have shown that competitive interactions dominate within industrially relevant brewery biofilm models and that antimicrobial treatment with sulfathiazole, if incomplete, can reduce the level of competition and therefore cause subsets of species to bloom, ultimately leading to enhanced overall tolerance. In addition, we have shown that the presence of competitors can also directly enhance the inherent tolerance to antimicrobials by driving specific tolerance phenotypes. Overall, our results emphasize that the increasingly recognized dominance of competition in multispecies biofilms is central to the enhanced antimicrobial tolerance and that antimicrobial activities against monoculture biofilms cannot predict their efficacy against multispecies biofilms. These insights should be taken into account when developing improved biofilm disinfection protocols for breweries specifically and industry and medicine in general. A possible solution is to alternate between different antimicrobials that target different species in order to avoid enrichment of specific species. In addition, our study underlines the need to further investigate and interfere with the mechanisms behind specific tolerance phenotypes that are driven by the presence of competitors.

Our analysis of the samples taken before and after CIP resulted in the identification of mainly harmless bacteria, belonging to the Pseudomonadaceae and Enterobacteriaceae. Consistently previous studies on contaminating brewery biofilms reported frequent occurrences of Acetobacteriaceae, Pseudomonadaceae, and Enterobacteriaceae, whereas beer spoilers were not often encountered [39, 43, 69-72]. It should be noted, however, that our analysis only included culturable species and could not distinguish between species that have the same morphology on the PCA plates. Nevertheless, harmless biofilms still cause visual slimy contaminations that deteriorate the general image of hygiene in the brewery, can cause corrosion and reduce process efficiency [39] and might act as primary colonizers that prime the surface for subsequent colonization by beer spoilers [38, 39].

Our data indicate that competitive interactions dominate among species within brewery biofilms, although interspecies competition is generally weaker than intra-specific competition. These data fit with a growing body of recent theoretic and experimental work indicating that competition, not cooperation, dominates interactions among microbial species. The genotypic view of social interactions predicts a low chance of evolution of cooperation between species, because this requires both a high within-genotype relatedness and sufficient niche separation to reduce ecological competition. Increased niche separation, however, often implies a decreased exchange of resources, which counteracts interactions, and further complicates the evolution of cooperation [23]. These predictions are confirmed by recent systematic screenings of inter-species interactions based on the cooperation criterion [22, 41, 73]. Also in these studies inter-species competition was found to be weaker than intra-specific competition [22, 40, 41, 73]. Moreover, Rivett et al. [41] showed that initially strong competitive interactions can weaken over time by divergence in resource use and increased niche complementarity. It should be noted that a number of studies did report a prevalence of positive interactions, however, these studies made use of alternative definitions. In a recent study, synergistic interactions were defined as the total amount of multispecies biofilm being higher than the sum of all monocultures and synergy was observed in $13 \%$ of the biofilms [74]. This definition is similar to the cooperation criterion, but since the effect of growth in coculture on the individual species is not included, the presence of cooperative interactions cannot be confirmed. In earlier studies, synergy required the total amount of multispecies biofilm to be higher than that of the best monoculture biofilm former and synergistic interactions were reported in, respectively, $11 \%, 63 \%$ and $30 \%$ of the biofilms [75-77]. Also here information on composition of the multispecies biofilm is needed to determine whether the described synergistic interactions are competitive or cooperative. It can, however, be deduced that these synergistic interactions are associated with a positive biodiversity effect, since both definitions imply the total amount of multispecies biofilm to be higher than the weighted average of the monocultures. Notably this positive biodiversity effect does imply niche complementarity, but can also partly be caused by positive selection effects. In conclusion, the importance of competition among species over cooperation is increasingly recognized and our data are consistent with this. However, an important note is that all studies described above, 
including ours, are based on culturable species, which might exclude species that are only able to grow in the presence of other species. Therefore, the prevalence of cooperation might be underestimated [22, 66].

The enhanced overall antimicrobial tolerance against sulfathiazole that we observed for each duo-species and multispecies biofilm model compared to the monoculture biofilms is consistent with the enhanced resistance found in the majority of multispecies biofilm studies [4-19, 78]. In most of these studies the enhanced tolerance was attributed to protective effects of the species on each other, however, generally without unraveling the mechanism of tolerance. In contrast, a minority of studies did not observe an effect of multispecies conditions on antimicrobial tolerance [79] or measured a decrease in tolerance in multispecies conditions [63, 80-83].

Our data suggest that the commonly observed enhanced antimicrobial tolerance of multispecies biofilms is associated with a reduction in the level of competition upon treatment, causing a subset of species to bloom. The dominance of competition among species over cooperation in untreated biofilms is, therefore, central to the enhanced antimicrobial tolerance. Indeed, incomplete inhibition of a network of cooperating species is expected, not to promote, but to pull down the remaining species because of abrogation of positive feedback loops, as is indicated by recent ecological network studies [84]. This would reduce, not increase, the overall tolerance of the multispecies biofilm [80]. Our models only provide examples of duo-species or multispecies biofilms in which specific species strongly suppress other species. Inhibition of the stronger competitors consequently reduces the competition that is experienced by the suppressed species and leads to an increased tolerance of the weaker competitors. However, the idea that antimicrobial tolerance in multispecies biofilms is connected to a reduction in competition should not be limited to this situation, as one can imagine that antimicrobial treatment can also reduce competition between equal competitors. For example, in the case of equally competing species that only produce their toxins when the population density of the other species is sufficiently high [85], a reduction of the population size by antimicrobial treatment would interfere with toxin production, reduce competition, and ultimately lead to increased antimicrobial tolerance compared to monoculture.

Based on the commonly found prevalence of competitive interactions within multispecies biofilms, it is expected that reduction in competition might often be the cause of increased tolerance. However, little is known about this because previous work mainly focused on characterizing the antimicrobial tolerance of mono-species and multispecies biofilms, without explicitly classifying the changes in interspecies interactions before and after treatment. In a number of studies, only the overall activity against the multispecies biofilm and the activity against the monocultures was measured, whereas information on individual species in coculture is essential to understand the inter-species interactions $[4,10$, $12,15,68]$. Similarly only determining the inhibition of each species in the coculture without looking at the effects in monoculture [80, 86-89], or only focusing on specific species within the multispecies biofilm $[6,7,14]$ does not allow to study all changes in inter-species interactions. Nevertheless, a few studies have been conducted in which the tolerance of each species was examined individually, both under monoculture and coculture biofilm conditions $[5,9,11$, $13,16-19,90]$. Whereas the data obtained would allow for a detailed analysis of the changes in inter-species interactions as proposed in this paper, this analysis is generally missing and the representation of the data in most cases did not allow us to interpret the data a posteriori. Nevertheless, one study on tolerance of a seven-species biofilm provided sufficient data and is consistent with our mechanism of "increased tolerance due to reduced competition" [90]. Some of the bacterial species experienced reduced growth due to competition in the untreated multispecies biofilm, whereas antimicrobial treatment restored their growth in the multispecies biofilm to the level of the treated monoculture biofilms. In contrast, but also consistent with our rationale, a reduction in antimicrobial tolerance under multispecies conditions has been explicitly associated with a reduction of (probably rare) cooperative inter-species interactions [80].

In our final model, we found that the presence of competitors can also directly enhance the inherent tolerance of other species by driving specific tolerance phenotypes. This could either be attributed to (i) protective effects of specific species on other species or to (ii) direct changes in tolerance phenotypes of specific species as a response to competitors. A previously described example of a protective effect occurs between competing Pseudomonas aeruginosa and Staphylococcus aureus species [78]. Respiration of S. aureus was found to be inhibited by a competitive interaction involving the exoproduct 4-hydroxy-2-heptylquinoline-Noxide of $P$. aeruginosa. As a consequence, aminoglycoside antibiotics were no longer taken up by $S$. aureus cells and their tolerance to these antibiotics increased. Furthermore, the presence of $P$. aeruginosa on a long term increased the production of highly resistant small-colony variants of $S$. aureus, which further improved the antimicrobial tolerance of $S$. aureus. In addition, it is becoming increasingly clear that bacteria can also directly sense the presence of competitors and respond appropriately (i.e., "competition sensing") [85]. Recent studies indicate that these responses can include upregulated biofilm formation [40], increased antibiotic, or toxin production [91-93], altered secretion of specific secondary metabolites [94], but also increased antibiotic tolerance ([91]; Roberfroid et al. personal communication). 
The insights discussed above, with regard to the increased tolerance of the biofilm models to sulfathiazole, can also be applied to provide an explanation for the insufficient removal of microbial contamination by CIP in the sampled breweries. In a number of samples, species that were isolated before CIP could not be retrieved after CIP, whereas the total species diversity and overall cell number in these samples increased. This suggests that CIP leads to a decrease in competition, similar to our observations in the first two biofilm models. Treatment with CIP likely removed the stronger competitors from the brewery biofilms, which allowed various weaker competitors to grow and ultimately resulted in an increase in community diversity and growth.

In conclusion, due to their commonly observed increased antimicrobial tolerance, multispecies biofilms remain challenging to eradicate. Accordingly we found multispecies biofilms to be a serious problem in breweries, as emphasized by the high microbial load of the isolated biofilm samples, both before and after CIP. An increased knowledge of the properties of these multispecies biofilms may aid to improve their control. Our study demonstrates that competitive inter-species interactions dominate within multispecies biofilms and have a strong influence on the outcome of antimicrobial treatment. Specifically we found that strongly suppressed species can bloom after inhibition of superior competitors by antimicrobial treatment, which results in increased tolerance and entails the risk of enrichment of specific beer spoilers. To avoid such unwanted effects of changing inter-species interactions, it would be useful to develop combination therapies that completely inhibit all species. Nevertheless, we also observed that the presence of competitors can increase the intrinsic tolerance of species by driving specific tolerance phenotypes. This means that antimicrobials that are completely effective against monoculture biofilms are not necessarily effective against the same species in coculture. Our study therefore underlines the need to further investigate and interfere with the mechanisms behind these specific tolerance phenotypes [95].

Acknowledgements We would like to thank S. Robijns, S. Van Puyvelde, and B. Lories for their valuable comments and for their assistance in sampling of brewery biofilms. We thank K. Deflem and D. De Coster for experimental assistance. This work was supported by the KU Leuven Research Fund (STG/16/022), by the Institute for the Promotion of Innovation through Science and Technology in Flanders under grant IWT-SBO 120050 (NEMOA) and by FWO-Vlaanderen (W0.009.16N). IP is a research assistant of the IWT-Vlaanderen (SB/ 131721). HPS acknowledges the receipt of a postdoctoral fellowship from FWO-Vlaanderen (PDO/11).

\section{Compliance with ethical standards}

Conflict of interest The authors declare that they have no conflict of interest.

\section{References}

1. Hall-Stoodley L, Costerton JW, Stoodley P. Bacterial biofilms: from the natural environment to infectious diseases. Nat Rev Microbiol. 2004;2:95-108.

2. Elias S, Banin E. Multi-species biofilms: living with friendly neighbors. FEMS Microbiol Rev. 2012;36:990-1004.

3. Stoodley P, Sauer K, Davies DG, Costerton JW. Biofilms as complex differentiated communities. Annu Rev Microbiol. 2002;56:187-209.

4. Adam B, Baillie GS, Douglas LJ. Mixed species biofilms of Candida albicans and Staphylococcus epidermidis. J Med Microbiol. 2002;51:344-9.

5. Harriott MM, Noverr MC. Candida albicans and Staphylococcus aureus form polymicrobial biofilms: effects on antimicrobial resistance. Antimicrob Agents Chemother. 2009;53:3914-22.

6. Jagmann N, Henke SF, Philipp B. Cells of Escherichia coli are protected against severe chemical stress by co-habiting cell aggregates formed by Pseudomonas aeruginosa. Appl Microbiol Biotechnol. 2015;99:8285-94.

7. Kumar A, Peng TY. Presence of Pseudomonas aeruginosa influences biofilm formation and surface protein expression of Staphylococcus aureus. Environ Microbiol. 2015;17:4459-68.

8. Lee KWK, Periasamy S, Mukherjee M, Xie C, Kjelleberg S, Rice SA. Biofilm development and enhanced stress resistance of a model, mixed-species community biofilm. ISME J. 2014;8:894-907.

9. Leriche V, Briandet R, Carpentier B. Ecology of mixed biofilms subjected daily to a chlorinated alkaline solution: spatial distribution of bacterial species suggests a protective effect of one species to another. Environ Microbiol. 2003;5:64-71.

10. Lopes SP, Ceri H, Azevedo NF, Pereira MO. Antibiotic resistance of mixed biofilms in cystic fibrosis: impact of emerging microorganisms on treatment of infection. Int $\mathbf{J}$ Antimicrob Agents. 2012;40:260-63.

11. Luppens SBI, Kara D, Bandounas L, Jonker MJ, Wittink FRa, Bruning O, et al. Effect of Veillonella parvula on the antimicrobial resistance and gene expression of Streptococcus mutans grown in a dual-species biofilm. Oral Microbiol Immunol. 2008;23:183-9.

12. Baffone W, Sorgente G, Campana R, Patrone V, Sisti D, Falcioni T. Comparative effect of chlorhexidine and some mouthrinses on bacterial biofilm formation on titanium surface. Curr Microbiol. 2011;62:445-51.

13. Schwering M, Song J, Louie M, Turner RJ, Ceri H. Multi-species biofilms defined from drinking water microorganisms provide increased protection against chlorine disinfection. Biofouling. 2013;29:917-28.

14. Shakeri S, Kermanshahi RK, Moghaddam MM, Emtiazi G. Assessment of biofilm cell removal and killing and biocide efficacy using the microtiter plate test. Biofouling. 2007;23:79-86.

15. Simões LC, Simões M, Vieira MJ. Influence of the diversity of bacterial isolates from drinking water on resistance of biofilms to disinfection. Appl Environ Microbiol. 2010;76:6673-79.

16. Simões M, Simões LC, Vieira MJ. Species association increases biofilm resistance to chemical and mechanical treatments. Water Res. 2009;43:229-37.

17. Van der Veen S, Abee T. Mixed species biofilms of Listeria monocytogenes and Lactobacillus plantarum show enhanced resistance to benzalkonium chloride and peracetic acid. Int J Food Microbiol. 2011;144:421-31.

18. Wang R, Kalchayanand N, Schmidt JW, Haray DM. Mixed biofilm formation by shiga toxin-producing Escherichia coli and Salmonella enterica serovar Typhimurium enhanced bacterial resistance to sanitization due to extracellular polymeric substances. J Food Prot. 2013;76:1513-22. 
19. Whiteley M, Ott JR, Weaver EA, McLean RJC. Effects of community composition and growth rate on aquifer biofilm bacteria and their susceptibility to betadine disinfection. Environ Microbiol. 2001;3:43-52.

20. Rakoff-Nahoum S, Foster KR, Comstock LE. The evolution of cooperation within the gut microbiota. Nature. 2016;533:255-59.

21. Harcombe W. Novel cooperation experimentally evolved between species. Evolution. 2010;64:2166-72.

22. Foster KR, Bell T. Competition, not cooperation, dominates interactions among culturable microbial species. Curr Biol. 2012;22:1845-50.

23. Mitri S, Foster KR. The genotypic view of social interactions in microbial communities. Annu Rev Genet. 2013;47:247-73.

24. West SA, Griffin AS, Gardner A. Social semantics: altruism, cooperation, mutualism, strong reciprocity and group selection. J Evol Biol. 2007;20:415-32.

25. Ghoul M, Mitri S. The ecology and evolution of microbial competition. Trends Microbiol. 2016;24:833-45.

26. Pfeiffer T, Schuster S, Bonhoeffer S. Cooperation and competition in the evolution of ATP-producing pathways. Science. 2001;292:504-07.

27. Scholz RL, Greenberg EP. Sociality in Escherichia coli: enterochelin is a private good at low cell density and can be shared at high cell density. J Bacteriol. 2015;197:2122-8.

28. Kim W, Racimo F, Schluter J, Levy SB, Foster KR. Importance of positioning for microbial evolution. Proc Natl Acad Sci USA. 2014;111:E1639-47.

29. Riley MA, Gordon DM. The ecological role of bacteriocins in bacterial competition. Trends Microbiol. 1999;7:129-33.

30. Lories B, Parijs I, Foster KR, Steenackers HP. Meeting report on the ASM conference on mechanisms of interbacterial cooperation and competition. J Bacteriol. 2017;199:e00403-17.

31. Russell AB, Peterson SB, Mougous JD. Type VI secretion system effectors: poisons with a purpose. Nat Rev Microbiol. 2014;12:137-48.

32. Cardinale BJ, Wright JP, Cadotte MW, Carroll IT, Hector A, Srivastava DS, et al. Impacts of plant diversity on biomass production increase through time because of species complementarity. Proc Natl Acad Sci USA. 2007;104:18123-8.

33. Loreau M, Hector A. Partitioning selection and complementarity in biodiversity experiments. Nature. 2001;412:72-6.

34. Morin X, Fahse L, Scherer-Lorenzen M, Bugmann H. Tree species richness promotes productivity in temperate forests through strong complementarity between species. Ecol Lett. 2011;14:1211-9.

35. Polley HW, Wilsey BJ, Derner JD. Do species evenness and plant density influence the magnitude of selection and complementarity effects in annual plant species mixtures? Ecol Lett. 2003;6:248-56.

36. Spehn EM, Hector A, Joshi J, Scherer-Lorenzen M, Schmid B, Bazeley-White E, et al. Ecosystem effects of biodiversity manipulatons in European grasslands. Ecol Monogr. 2005;75:37-63.

37. Maifreni M, Frigo F, Bartolomeoli I, Buiatti S, Picon S, Marino M. Bacterial biofilm as a possible source of contamination in the microbrewery environment. Food Control. 2015;50:809-14.

38. Mamvura T, Iyuke S. Soil films in the beverage industry: a review. J Inst Brew. 2011;117:608-16.

39. Storgards E, Tapani K. Microbial attachment and biofilm formation in brewery bottling plants. Am Soc Brew Chem. 2006;64:8-15.

40. Oliveira NM, Martinez-Garcia E, Xavier J, Durham WM, Kolter $\mathrm{R}$, Kim W, et al. Biofilm formation as a response to ecological competition. PLoS Biol. 2015;13:1-23.

41. Rivett DW, Scheuerl T, Culbert CT, Mombrikotb SB, Johnstone E, Barraclough TG, et al. Resource-dependent attenuation of species interactions during bacterial succession. ISME J. 2016;10:2259-68.

42. Chen L, Chen R, Yin H, Sui J, Lin H. Cleaning in place with onsite-generated electrolysed oxidizing water for water-saving disinfection in breweries. J Inst Brew. 2012;118:401-05.

43. Timke M, Wang-Lieu N. Community structure and diversity of biofilms from a beer bottling plant as revealed using 16S rRNA gene clone libraries. Appl Environ Microbiol. 2005;71: 6446-52.

44. Cai H, Archambault M, Prescott JF. 16S ribosomal RNA sequence-based identification of veterinary clinical bacteria. J Vet Diagn Investig. 2003;15:465-9.

45. White TJ, Bruns T, Lee S, Taylor JW. Amplification and direct sequencing of fungal ribosomal RNA genes for phylogenetics. Pp. 315-322 In: PCR Protocols: A Guide to Methods and Applications, eds. Innis, M. A., D. H. Gelfand, J. J. Sninsky, and T. J. White. Academic Press, Inc., New York. (1990)

46. Ceri H, Olson ME, Stremick C, Read RR, Morck D, Buret A. The Calgary biofilm device: new technology for rapid determination of antibiotic susceptibilities of bacterial biofilms. J Clin Microbiol. 1999;37:1771-6.

47. Opperman TJ, Kwasny SM, Williams JD, Khan AR, Peet NP, Moir DT, et al. Aryl rhodanines specifically inhibit staphylococcal and enterococcal biofilm formation. Antimicrob Agents Chemother. 2009;53:4357-67.

48. Barraud N, Hassett DJ, Hwang S-H, Rice SA, Kjelleberg S, Webb JS. Involvement of nitric oxide in biofilm dispersal of Pseudomonas aeruginosa. J Bacteriol. 2006;188:7344-53.

49. Nithya C, Devi MG, Karutha Pandian S. A novel compound from the marine bacterium Bacillus pumilus S6-15 inhibits biofilm formation in gram-positive and gram-negative species. Biofouling. 2011;27:519-28.

50. Lynch AS, Abbanat D. New antibiotic agents and approaches to treat biofilm-associated infections. Expert Opin Ther Pat. 2010;20:1373-87.

51. Antoniani D, Bocci P, Maciag A, Raffaelli N, Landini P. Monitoring of diguanylate cyclase activity and of cyclic-di-GMP biosynthesis by whole-cell assays suitable for high-throughput screening of biofilm inhibitors. Appl Microbiol Biotechnol. 2010;85:1095-104.

52. Cotter PA, Stibitz S. c-di-GMP-mediated regulation of virulence and biofilm formation. Curr Opin Microbiol. 2007;10:17-23.

53. Hengge R. Principles of c-di-GMP signalling in bacteria. Nat Rev Microbiol. 2009;7:263-73.

54. Romling U, Galperin MY, Gomelsky M. Cyclic di-GMP: the first 25 years of a universal bacterial second messenger. Microbiol Mol Biol Rev. 2013;77:1-52.

55. Fox JW. Interpreting the 'selection effect' of biodiversity on ecosystem function. Ecol Lett. 2005;8:846-56.

56. Loreau M. Biodiversity and ecosystem functioning: recent theoretical advances. Oikos. 2000;91:3-17.

57. Turnbull LA, Levine JM, Loreau M, Hector A. Coexistence, niches and biodiversity effects on ecosystem functioning. Ecol Lett. 2013;16:116-27.

58. Loreau M, de Mazancourt C. Biodiversity and ecosystem stability: a synthesis of underlying mechanisms. Ecol Lett. 2013;16:106-15.

59. Alley TR. Competition theory, evolution, and the concept of an ecological niche. Acta Biotheor. 1982;31:165-79.

60. Hardin G. The competitive exclusion principle. Science. 1960;131:1292-7.

61. Mozina SS, Klancknik A, Raspor P. Mechanisms of microbial resistance in biofilms. Biofilms in Bioengineering, Edition: First, Chapter: Chapter 12. Mechanisms of Microbial Resistance in Biofilms, Publisher: Nova Science Publishers, Editors: Manuel Simões, Filipe Mergulhão, 311-332. 
62. Burmølle M, Ren D, Bjarnsholt T, Sørensen SJ. Interactions in multispecies biofilms: do they actually matter? Trends Microbiol. 2014;22:84-91.

63. Chorianopoulos NG, Giaouris ED, Skandamis PN, Haroutounian SA, Nychas GJE. Disinfectant test against monoculture and mixed-culture biofilms composed of technological, spoilage and pathogenic bacteria: bactericidal effect of essential oil and hydrosol of Satureja thymbra and comparison with standard acidbase sanitizers. J Appl Microbiol. 2008;104:1586-96.

64. Giaouris E, Chorianopoulos N, Doulgeraki A, Nychas GJ. Coculture with Listeria monocytogenes within a dual-species biofilm community strongly increases resistance of Pseudomonas putida to benzalkonium chloride. PLoS One. 2013;8:1-14.

65. Kostaki M, Chorianopoulos N, Braxou E, Nychas GJ, Giaouris E. Differential biofilm formation and chemical disinfection resistance of sessile cells of Listeria monocytogenes strains under monospecies and dual-species (with Salmonella enterica) conditions. Appl Environ Microbiol. 2012;78:2586-95.

66. Røder HL, Sørensen SJ, Burmølle M. Studying bacterial multispecies biofilms: where to start? Trends Microbiol. 2016;24:503-13.

67. Tan CH, Kelvin Lee KW, Burmølle M, Kjelleberg S, Rice SA, Lee KWK, et al. All together now: experimental multispecies biofilm model systems. Environ Microbiol. 2016;13:385-92.

68. Burmølle M, Webb JS, Rao D, Hansen LH, Sørensen SJ, Kjelleberg S. Enhanced biofilm formation and increased resistance to antimicrobial agents and bacterial invasion are caused by synergistic interactions in multispecies biofilms. Appl Environ Microbiol. 2006;72:3916-23.

69. Priha O, Raulio M, Cooke K, Fisher L, Hill C, Hylkinen S, et al. Microbial populations on brewery filling hall surfaces-Progress towards functional coatings. Food Control. 2015;55:1-11.

70. Priha O, Raulio M, Maukonen J, Vehviläinen A-K, Storgårds E. Bacterial populations on brewery filling hall surfaces as revealed by next-generation sequencing. Biofouling. 2016;32:571-81.

71. Quain D, Storgårds E. The extraordinary world of biofilms. Brew Distill Int. 2009;5:31-3.

72. Timke M, Wolking D, Wang-Lieu NQ, Altendorf K, Lipski A. Microbial composition of biofilms in a brewery investigated by fatty acid analysis, fluorescence in situ hybridisation and isolation techniques. Appl Microbiol Biotechnol. 2004;66:100-7.

73. Fiegna F, Moreno-Letelier A, Bell T, Barraclough TG. Evolution of species interactions determines microbial community productivity in new environments. ISME J. 2015;9:1235-45.

74. Madsen JS, Røder HL, Russel J, Sørensen H, Burmølle M, Sørensen SJ. Coexistence facilitates interspecific biofilm formation in complex microbial communities. Environ Microbiol. 2016;18:2565-74.

75. Ren D, Madsen JS, Sørensen SJ, Burmølle M. High prevalence of biofilm synergy among bacterial soil isolates in cocultures indicates bacterial interspecific cooperation. ISME J. 2015;9:81-9.

76. Burmølle M, Hansen LH, Sørensen SJ. Establishment and early succession of a multispecies biofilm composed of soil bacteria. Microb Ecol. 2007;54:352-62.

77. Røder HL, Raghupathi PK, Herschend J, Brejnrod A, Knøchel S, Sørensen SJ, et al. Interspecies interactions result in enhanced biofilm formation by co-cultures of bacteria isolated from a food processing environment. Food Microbiol. 2015;51:18-24.

78. Hoffman LR, Déziel E, D'Argenio DA, Lépine F, Emerson J, McNamara S, et al. Selection for Staphylococcus aureus smallcolony variants due to growth in the presence of Pseudomonas aeruginosa. Proc Natl Acad Sci USA. 2006;103:19890-5.
79. Gkana EN, Giaouris ED, Doulgeraki AI, Kathariou S, Nychas GJE. Biofilm formation by Salmonella Typhimurium and Staphylococcus aureus on stainless steel under either mono- or dualspecies multi-strain conditions and resistance of sessile communities to sub-lethal chemical disinfection. Food Control. 2017;73:838-46.

80. Feldman M, Ginsburg I, Al-Quntar A, Steinberg D. Thiazolidinedione-8 alters symbiotic relationship in C. albicans-S. mutans dual species biofilm. Front Microbiol. 2016;7:140.

81. Kart D, Tavernier S, Van Acker H, Nelis HJ, Coenye T. Activity of disinfectants against multispecies biofilms formed by Staphylococcus aureus, Candida albicans and Pseudomonas aeruginosa. Biofouling. 2014;30:377-83.

82. Lindsay D, Brözel VS, Mostert JF, Von Holy A. Differential efficacy of a chlorine dioxide-containing sanitizer against single species and binary biofilms of a dairy-associated Bacillus cereus and a Pseudomonas fluorescens isolate. J Appl Microbiol. 2002;92:352-61.

83. Yassin SA, German MJ, Rolland SL, Rickard AH, Jakubovics NS. Inhibition of multispecies biofilms by a fluoride-releasing dental prosthesis copolymer. J Dent. 2016;48:62-70.

84. Coyte KZ, Schluter J, Foster KR. The ecology of the microbiome: networks, competition, and stability. Science. 2015;350:663-6.

85. Cornforth DM, Foster KR. Competition sensing: the social side of bacterial stress responses. Nat Rev Microbiol. 2013;11:285-93.

86. DeLeon S, Clinton A, Fowler H, Everett J, Horswill AR, Rumbaugh KP. Synergistic interactions of Pseudomonas aeruginosa and Staphylococcus aureus in an in vitro wound model. Infect Immun. 2014;82:4718-28.

87. Hill KE, Malic S, McKee R, Rennison T, Harding KG, Williams DW, et al. An in vitro model of chronic wound biofilms to test wound dressings and assess antimicrobial susceptibilities. J Antimicrob Chemother. 2010;65:1195-206.

88. Norwood DE, Gilmour A. The growth and resistance to sodium hypochlorite of Listeria monocytogenes in a steady-state multispecies biofilm. J Appl Microbiol. 2000;88:512-20.

89. Sun Y, Dowd SE, Smith E, Rhoads DD, Wolcott RD. In vitro multispecies Lubbock chronic wound biofilm model. Wound Repair Regen. 2008;16:805-13.

90. Elvers KT, Leeming K, Lappin-Scott HM. Binary and mixed population biofilms: time-lapse image analysis and disinfection with biocides. J Ind Microbiol Biotechnol. 2002;29:331-8.

91. Abrudan MI, Smakman F, Grimbergen AJ, Westhoff S, Miller EL, van Wezel GP, et al. Socially mediated induction and suppression of antibiosis during bacterial coexistence. Proc Natl Acad Sci USA. 2015;112:11054-9.

92. Rosenberg G, Steinberg N, Oppenheimer-shaanan Y, Olender T, Doron S, Ben-ari J. Not so simple, not so subtle: the interspecies competition between Bacillus simplex and Bacillus subtilis and its impact on the evolution of biofilms. NPJ Biofilms Microb. 2016;2:1-11.

93. Le Roux M, Kirkpatrick RL, Montauti EI, Tran BQ, Brook Peterson S, Harding BN, et al. Kin cell lysis is a danger signal that activates antibacterial pathways of Pseudomonas aeruginosa. Elife. 2015;2015:1-65.

94. Traxler MF, Watrous JD, Alexandrov T, Dorrestein PC, Kolter R. Interspecies interactions stimulate diversification of the Streptomyces coelicolor secreted metabolome. MBio. 2013;4:1-12.

95. Kelsic ED, Zhao J, Vetsigian K, Kishony R. Counteraction of antibiotic production and degradation stabilizes microbial communities. Nature. 2015;521:516-9. 